\title{
PERFIL DERMATOGLÍFICO E QUALIDADES FÍSICAS BÁSICAS DE JOVENS ATLETAS
} DE VOLEIBOL

Hermógenes Gomes Silva

Cícero Luciano Alves Costa

Hudday Mendes da Silva

Richardson Dylsen de Souza Capistrano

\section{Resumo}

O objetivo do estudo é identificar o perfil dermatoglífico e o nível das qualidades físicas básicas (QFB) de jovens atletas de voleibol de Juazeiro do Norte. A amostra foi composta por 12 jovens atletas de voleibol. Utilizou-se o Método Dermatoglífico para identificação do perfil genético e a bateria do PROESP-BR. A dermatoglifia apontou a velocidade sendo a QFB com maior predisposição, porém, o teste de correlação de Spearman não apontou fortes associações entre os índices dermatoglíficos e as QFB. Os sujeitos apresentaram resultados inferiores a estudos realizados com atletas da mesma categoria. A característica amadora do esporte na região pode justificar tal discrepância, pois a performance esportiva não depende somente de fatores hereditários como seus determinantes, mas também, de fatores fenotípicos.

\section{Palavras-Chave}

Perfil Dermatoglífico; Qualidades Físicas Básicas; Voleibol.

\section{DERMATOGLYPHIC PROFILE AND BASIC PHYSICAL QUALITIES OF YOUNG VOLLEYBALL ATHLETES}

Hermógenes Gomes Silva

Cícero Luciano Alves Costa

Hudday Mendes da Silva

Richardson Dylsen de Souza Capistrano

\begin{abstract}
The objective of the study is to identify the dermatoglyphic profile and level of basic physical qualities (QFB) of young volleyball athletes from Juazeiro do Norte. The sample was composed for 12 young volleyball athletes. It was used the Dermatoglyphic method to identify the genetic profile of the subjects, and the PROESP-BR battery. The dermatoglyphic showed the speed being QFB with higher predisposition, however, the Spearman correlation test does not showed strong associations between dermatoglyphic indexes and the QFB.The individuals showed results lower than studies made with athletes of the same category. The amateur characteristic of the Sport in the region can justify such discrepancy,because the Sport performance does not depend only of hereditary factors as its determinants, but also, of phenotypic factors.
\end{abstract}

\section{Key-Words}

Profile Dermatoglyphic; Basic physical qualities; Volleyball. 


\section{INTRODUÇÃO}

No voleibol, algumas características cineantropométricas, tais como a estatura e comprimento de membros, juntamente com outras ligadas ao desempenho motor, como o salto vertical e agilidade são bastante utilizadas na identificação do perfil de atletas e na alta performance esportiva (FILIN; VOLKOV, 1998; DANTAS, 1998; MASSA, et al., 2003; DUNCAN, et al., 2005; GABBETT; GEORGIEFF, 2007). Entretanto, os instrumentos utilizados para a identificação de talentos de forma eficaz ainda não são devidamente conhecidos (RÉ et al., 2003).

Segundo Del Villar (1992), a constituição física é resultante de características genéticas hereditárias nas quais se identificando podem ser utilizadas no direcionamento para um determinado tipo de modalidade esportiva. Desta forma, a dermatoglifia tem se mostrado uma importante ferramenta na identificação do perfil de atletas, pois leva em conta aspectos de predisposição genética para o desenvolvimento das qualidades físicas básicas (SILVA DANTAS; FERNANDES FILHO, 2002).

Estudos são desenvolvidos no Brasil com o intuito de identificar o perfil dos atletas de alto rendimento de diversas modalidades esportivas, porém, pouco tem se preocupado a identificar tais características em desportistas amadores ou de categorias de base (JOÃO; FERNANDES; FILHO, 2002; MEDINA; FERNANDES FILHO, 2002; SILVA DANTAS; FERNANDES FILHO, 2002; CASTANHEDE, et al., 2003; SILVA DANTAS; ALONSO; FERNANDES FILHO, 2004; PAVEL; FERNANDES FILHO, 2004; MEDEIROS; ROCHA; FERNANDES FILHO, 2005; CUNHA JÚNIOR; FERNANDES FILHO, 2005; TUCHE, et al. 2005; CUNHA JÚNIOR, et al. 2006; FONSECA, et al., 2008).

Segundo Tubino e Dacosta (2008), a ciência esportiva tem se apoiado na pesquisa genética fazendo uso de códigos hereditários na identificação de talentos e na individualização do treinamento. Assim, a dermatoglifia tem sido um dos principais instrumentos, pois utiliza as impressões digitais como marcadores genéticos na identificação das qualidades físicas básicas do indivíduo (SILVA DANTAS; FERNANDES FILHO, 2002; FERNANDES FILHO; SILVA DANTAS; ROQUETTI FERNANDES, 2006).

Baseados nas pesquisas de Antropologia, morfologia e Genética Esportiva, do Instituto de Verificação Científica da Cultura Física da Rússia (VNIIFK) - Moscou, Fernandes Filho e Abramova referenciam que o Método Dermatoglífico é usado diretamente na seleção esportiva, correlacionando-se com as qualidades físicas básicas (ROLIM FILHO; FERNANDES FILHO, 2007). Os desenhos formados pelas 
impressões digitais constituem uma característica qualitativa a partir de três grupos de desenhos: arco (A), presilha (L) e verticílio (W). Enquanto isso, a característica quantitativa é representada pela quantidade de linhas dentro do padrão dermatoglífico (ALONSO; SILVA DANTAS, 2008). Além da análise do padrão e da quantidade de linhas, também é feito o cálculo do índice delta (D10), pela soma da presença dos trirrádios de todos os desenhos (FERNANDES FILHO, 1997; SILVA DANTAS; FERNANDES FILHO, 2002; SILVA DANTAS; ALONSO; FERNANDES FILHO, 2004).

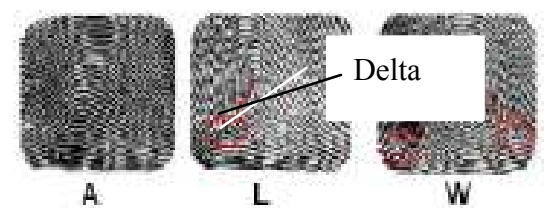

Figura 1 - Tipos de desenhos das impressões digitais

No arco não existe presença de trirrádio, na presilha encontra-se um trirrádio, e no verticílio dois trirrádios, evidenciando que quanto mais trirrádios se encontrem, maior é a complexidade do desenho (SILVA DANTAS; FERNANDES FILHO, 2002). Segundo a literatura vigente (ABRAMOVA, et al. 2005; MEDEIROS, et al., 2005; CUNHA; FERNANDES FILHO, 2005) a qualidade força pura é identificada pela presença de $\operatorname{arcos}(\mathrm{A})$; velocidade pura, pela presença de presilhas (L); e as qualidades físicas de resistência e coordenação, pela presença de verticílios (W).

O somatório da quantidade total de linhas (SQTL) é feito a partir da contagem das linhas existentes entre o centro do desenho e o delta. A alta intensidade do SQTL representa maior capacidade predisposição à resistência e coordenação, o mesmo acontecendo com a grande presença de deltas (D10). Do contrário, as baixas intensidades destes índices implicam numa maior predisposição ao desenvolvimento de força, velocidade e potência (FERNANDES FILHO; SILVA DANTAS; ROQUETTI FERNANDES, 2006).

Baseado nestes pressupostos, o objetivo geral deste estudo é identificar o perfil de jovens atletas de voleibol da cidade de Juazeiro do Norte, analisando o nível das qualidades físicas básicas e relacionando às possíveis influências da predisposição genética apontada pelo método dermatoglífico.

\section{MATERIAIS E MÉTODOS}

$\mathrm{O}$ estudo se caracteriza como sendo descritivo, quantitativo, com perfil e delineamento ex post facto (THOMAS; NELSON, 2002). A amostra foi intencional, composta por 12 indivíduos do gênero masculino, sendo todos participantes da equipe de voleibol do CEFET - CE (Centro Federal de Educação Tecnológica) da cidade de Juazeiro do Norte com média de 16,08 $\pm 0,79$ dp anos de idade. O treinador da 
$\overline{\text { equipe foi contatado para exposição dos objetivos do trabalho e posteriormente em uma reunião com } o}$ time, todos os componentes também foram devidamente informados e convidados a participarem da pesquisa. Os pais ou responsáveis dos adolescentes assinaram o Termo de Consentimento Livre e Esclarecido (TCLE). Esta pesquisa foi aprovada pelo Comitê de Ética e Pesquisa da Universidade Castelo Branco (UCB-RJ), sob o protocolo 0017/2009 (UCB/VREPGPE/COMEP/PROCIMH).

Para análise das qualidades físicas básicas foram usados os seguintes testes propostos pela bateria do Projeto Esporte Brasil, PROESP-BR (GAYA, A; SILVA, G, 2007): teste de sentar e alcançar para mensurar a flexibilidade; teste de abdominais sit up's para RML; teste do quadrado para agilidade; corrida de 20 metros para avaliar a velocidade; e o teste de corrida e caminhada em 09 minutos para estimativa da resistência aeróbia. Após a aplicação da bateria de testes, todos os resultados obtidos foram classificados a partir das tabelas normativas do PROESP-BR. Além dos testes da bateria PROESP-BR, utilizou-se os testes Impulsão Vertical (PITANGA, 2004) e o teste de máxima preensão manual direita e esquerda através do dinamômetro digital com precisão de $0,1 \mathrm{~kg} / \mathrm{f}$.

Para a variável antropométrica foram mensuradas as dobras cutâneas triciptal (TR) e subescapular (SE) com um adipômetro Holtain com resolução de $0,1 \mathrm{~mm}$. Para a estimativa do percentual de gordura foi utilizada a equação proposta por Boileau et al. citado por Petroski (2007) para jovens de 7 a 17 anos, sendo os resultados classificados a partir do critério de referência de Lohman (1992). As dobras cutâneas TR e SE são utilizadas na investigação da composição corporal de crianças e adolescentes em diversos estudos realizados no Brasil (GUEDES; GUEDES, 1997; BOELHOUWER; BORGES, 2002; GUEDES, et al., 2002; SILVA, 2002; RONQUE, 2003; MOREIRA et al., 2005; GONÇALVES, et al., 2007).

O protocolo escolhido para analisar o potencial genético através das impressões digitais, foi o Método Dermatoglífico de Cummins e Midlo (1942), sendo este um instrumento validado e bastante usado no Brasil em pesquisas referentes ao tema. Para tanto foi utilizado papel de rugosidade e densidade médias (papel Ofício A4), além de uma almofada especial para impressões digitais. Após a obtenção das impressões, foi realizado o processo de leitura dos tipos de desenhos, o cálculo do índice delta (D10) e do somatório da quantidade total de linhas (SQTL).

Após a coleta e classificação dos dados foi organizado um banco no programa Microsoft Office Excel 2003. Foi utilizada estatística descritiva de média e desvio padrão para apresentação dos resultados dos índices dermatoglíficos e das qualidades físicas básicas. Além disso utilizou-se distribuição de freqüência 
para analisar o atendimento aos critérios de referência do PROESP-BR. Ainda com o intuito de analisar o perfil, foi confeccionado o gráfico "radar Fernandes Filho" com o perfil total dos valores normatizados. A análise inferencial se deu com o teste de correlação de Spearman para verificar possíveis associações entre os índices dermatoglíficos e o resultado dos testes motores. O nível de significância adotado foi de $5 \%$.

\section{Análise e discussão dos resultados}

As características digitais apontadas pelo método dermatoglífico nos fornecem importantes informações acerca da predisposição ao desenvolvimento das qualidades físicas básica. $\mathrm{O}$ somatório da quantidade total de linhas (SQTL) é influenciado diretamente pela quantidade de linhas de cada dedo (CUNHA JÚNIOR; FERNANDES FILHO, 2006), sendo as médias do somatório da quantidade de linhas dos dedos da mão esquerda (MESQL) e do somatório da quantidade de linhas dos dedos da mão direita (MDSQL) encontrados neste estudo considerados baixos quando comparados a esportista de alto rendimento.

TABELA 1 - Valores médios e desvio padrão para a quantidade de linhas de cada dedo

\begin{tabular}{lcccc}
\hline & Média & min_média & máx_média & Desvio padrão \\
\hline MESQL1 & 11,0 & 7,1 & 14,8 & 6,7 \\
MESQL2 & 7,2 & 4,0 & 10,4 & 5,6 \\
MESQL3 & 7,7 & 4,2 & 11,3 & 6,2 \\
MESQL4 & 11,1 & 7,9 & 14,3 & 5,6 \\
MESQL5 & 8,4 & 5,5 & 11,3 & 5,0 \\
MDSQL1 & 14,3 & 11,2 & 17,4 & 5,4 \\
MDSQL2 & 7,6 & 3,9 & 11,4 & 6,6 \\
MDSQL3 & 8,1 & 6,0 & 10,3 & 3,8 \\
MDSQL4 & 9,5 & 6,7 & 12,4 & 5,0 \\
MDSQL5 & 9,4 & 6,7 & 12,1 & 4,7 \\
\hline
\end{tabular}

Quanto ao comportamento de aumento e diminuição da quantidade de linhas da mão esquerda e direita, o estudo de Fonseca et al. (2008) com atletas da seleção brasileira feminina de voleibol se assemelha com o presente estudo no que diz respeito à maior quantidade de linhas do $1^{\circ}$ dedo da mão direita que é maior do que da esquerda, assim como ocorre um decréscimo da quantidade de linhas para o $2^{\circ}$ dedo de ambas as mãos (Figura 1). 
Figura 2 - Particularidades do somatório da quantidade de linhas da mão esquerda (MESQL) e direita (MDSQL)

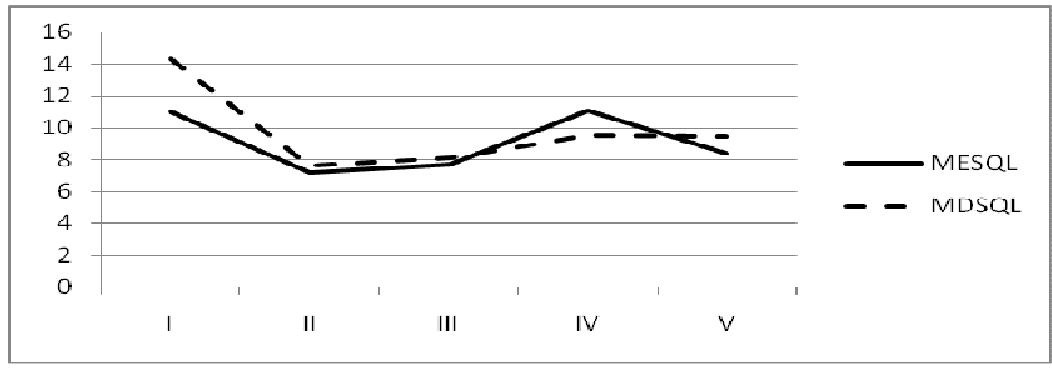

$\mathrm{Na}$ Tabela 2, são apontados os valores médios e desvio padrão da quantidade de arcos (A), presilhas (L), verticílios (W), índice delta D10 e SQTL. Pode-se observar que o tipo de desenho mais constante nas impressões digitais dos jovens atletas é a presilha (L), seguido pelo verticílio (W) e com uma menor quantidade de arcos (A). Além disso, foram encontrados valores considerados baixos de índice delta (D10) e do somatório da quantidade total de linhas (SQTL).

TABELA 2 - Valores médios e desvio padrão dos tipos de desenhos A, L, W, índice delta D10 e SQTL encontrados nas impressões digitais.

\begin{tabular}{ccccc}
\hline & Média & min_média & máx_média & $\begin{array}{c}\text { Desvio } \\
\text { padrão }\end{array}$ \\
\hline A & 1,1 & 0,1 & 2,1 & 1,7 \\
L & 7,3 & 5,9 & 8,6 & 2,4 \\
W & 1,7 & 0,2 & 3,1 & 2,5 \\
D10 & 10,6 & 8,6 & 12,6 & 3,5 \\
SQTL & 94,3 & 69,8 & 118,9 & 43,3 \\
\hline
\end{tabular}

A maior presença de L, e os baixos índices de W, D10 e SQTL, são indicadores de que os atletas investigados possuem uma maior predisposição ao desenvolvimento da qualidade física velocidade, pois, segundo a literatura, estas características são próprias das modalidades de alta potência e tempo curto de realização (FERNANDES FILHO; SILVA DANTAS, 2002; ROQUETTI FERNANDES, 2006).

Tais índices, além de ficarem bem abaixo dos encontrados em atletas de alto rendimento, quando comparados com os atletas da seleção brasileira infanto-juvenil (ZARY; FERNANDES FILHO, 2007), também aparecem com valores menores. Tanto no que diz respeito ao D10 quanto ao SQTL. Ainda mais, a média de L do citado estudo foi de 5,9, de W 3,3 e A de 0,8. Ou seja, segundo a literatura vigente no 
tema (ABRAMOVA et al. 1995), os atletas investigados no presente estudo tem uma menor predisposição ao desenvolvimento das qualidades físicas de resistência e coordenação quando comparados com os atletas da seleção infanto-juvenil e adulto.

TABELA 3 - Comparação dos índices dermatoglíficos entre os atletas da seleção nacional adulto e infanto-juvenil masculina de voleibol com o presente estudo

\begin{tabular}{cccccc}
\hline CATEGORIA & $\mathrm{A}$ & $\mathrm{L}$ & $\mathrm{W}$ & $\mathrm{D} 10$ & SQTL \\
\hline Adulto & $0,3 \pm 0,7$ & $5,8 \pm 2,2$ & $3,8 \pm 2,4$ & $13,5 \pm 2,8$ & $132,0 \pm 38,2$ \\
Infanto-Juvenil & $0,8 \pm 1,4$ & $5,9 \pm 2,1$ & $3,3 \pm 2,6$ & $11,9 \pm 3,5$ & $118,9 \pm 43,8$ \\
Presente & $1,1 \pm 1,7$ & $7,3 \pm 2,4$ & $1,7 \pm 2,5$ & $10,6 \pm 3,5$ & $94,3 \pm 43,3$ \\
Estudo & & & & & \\
\hline
\end{tabular}

A análise das fórmulas digitais nos fornece informações importantes acerca das qualidades físicas e as derivações propensas ao desenvolvimento no indivíduo. Na tabela 3 pode-se observar que as fórmulas digitais mais presentes nos jovens atletas investigados foi $\mathrm{L}>\mathrm{W}(33,3 \%)$, seguida de AL (25\%). Esta associação nos permite inferir que a velocidade continua sendo a qualidade física com maior predisposição genética encontrada no grupo, porém, a associação com os demais desenhos também demonstra predisposição a velocidade resistente e força potência.

TABELA 4 - Distribuição de frequência das fórmulas digitais

\begin{tabular}{llllllll}
\hline $\mathbf{1 0}^{\mathbf{a}}$ & $\mathbf{A L}$ & $\mathbf{A L W}$ & $\mathbf{1 0 L}$ & $\mathbf{L}=\mathbf{W}$ & $\mathbf{1 0 W}$ & $\mathbf{L}>\mathbf{W}$ & $\mathbf{W}>\mathbf{L}$ \\
\hline $0,0 \%$ & $25,0 \%$ & $16,7 \%$ & $16,7 \%$ & $0,0 \%$ & $0,0 \%$ & $33,3 \%$ & $8,3 \%$ \\
\hline
\end{tabular}

A seguir, na tabela 04, são apresentados os valores médios, e desvio padrão dos resultados obtidos pelo grupo nos testes escolhidos para avaliar as características somatomotoras.

TABELA 5 - Valores médios e desvio padrão para estatura, percentual de gordura e testes de aptidão $(\mathrm{n}=12)$

\begin{tabular}{ccccc}
\hline & min_média & média & máx_média & Dp \\
\hline Idade & 15,63 & 16,08 & 16,53 & 0,79 \\
Estatura & 170,87 & 175,67 & 180,46 & 8,48 \\
\% de Gordura & 15,07 & 16,84 & 18,61 & 3,13 \\
Res.Aeróbia (m) & 1711,84 & 1794,67 & 1877,49 & 146,39 \\
Agilidade (s) & 5,28 & 5,42 & 5,56 & 0,25 \\
RML (r) & 30,59 & 33,67 & 36,74 & 5,43 \\
Flexibilidade (cm) & 19,38 & 24,26 & 29,13 & 8,62 \\
Velocidade (s) & 3,39 & 3,49 & 3,60 & 0,19 \\
PMD (kg/f) & 42,6 & 37,96 & 47,16 & 8,13 \\
PME (kg/f) & 39,8 & 36,07 & 43,59 & 6,64 \\
Força/potência (cm) & 34,88 & 38,17 & 41,47 & 5,82 \\
\hline
\end{tabular}


Em relação ao percentual de gordura dos jovens atletas de voleibol, a média encontrada está dentro do padrão de normalidade referenciado por Lohman (1992) para crianças e adolescentes. Apenas 8,3\% se encontram com o percentual de gordura moderadamente alto para a idade. Mesmo assim, a maioria dos avaliados se encontram com a estimativa do percentual de gordura adequado, obtendo uma classificação ótima, o que pode promover um melhor desempenho já que a baixa ingestão de alimentos, principalmente de carboidratos, pode ocasionar deficiências energéticas, comprometendo o desempenho e a qualidade de vida. Ainda assim, a média do percentual de gordura apresentou-se superior aos valores da seleção brasileira infanto-juvenil (11,0 $\pm 2,3 \mathrm{dp})$, como também acima das médias das equipes dos estados do Amazonas e do Sergipe, com 13,2 $\pm 5,8 \mathrm{dp}$ e 12,3 \pm 4,7 dp, respectivamente (CABRAL, et al., 2005).

Os valores obtidos a partir dos testes da bateria PROESP-BR, quando classificados a partir das tabelas normativas apontam os seguintes resultados: para a variável de preensão manual direita e esquerda, a maioria dos sujeitos obteve uma classificação muito fraca, já para o teste de abdominais em um minuto todos os avaliados não conseguiram atender aos critérios de referências estando classificadas em muito fraco, fraco e razoável. Quanto ao teste de agilidade os resultados foram satisfatórios com $91,6 \%$ da amostra sendo classificado como bom e muito bom e apenas $8,3 \%$ como razoável. Para o teste de velocidade apresentaram-se resultados razoáveis com 58,3\% dos avaliados e apenas $16,7 \%$ tendo resultados bons, para o restante a classificação foi de fraco a muito fraco. Em relação ao teste de Resistência Aeróbia 75\% dos sujeitos obtiveram resultados entre bom e muito bom, enquanto isso, apenas $25 \%$ registrou índices razoáveis.

No trabalho de Massa et al. (2003), onde aplicava junto a equipes de voleibol masculino alguns testes para informações sobre descobertas de talentos, no teste de impulsão vertical com auxilio dos membros inferiores, os resultados obtidos foram de $55,5 \pm 5,7 \mathrm{~cm}$ para a equipe infanto-juvenil, enquanto que para o presente estudo o valor médio obtido foi de $38,2 \pm 5,8 \mathrm{~cm}$ havendo uma diferença de aproximadamente 17 $\mathrm{cm}$ em relação ao estudo citado. Esta média também está abaixo do resultado obtido por jovens atletas de basquete $(48,34 \pm 16,56 \mathrm{~cm})$, e de futebol $(41,67 \pm 18,15 \mathrm{~cm})$ (GONÇALVES et al., 2007). Já no estudo de Heimer e colaboradores com jogadores da antiga Iuguslávia, verificou-se valores de $64,2 \mathrm{~cm}$ no teste de impulsão vertical (MASSA et al., 2003).

Em um estudo realizado no Reino Unido com jovens atletas de Voleibol com média de idade de 17,5 \pm 0,5 dp anos, foram pesquisadas algumas variáveis intervenientes na performance deste esporte comparando os resultados entre os atletas que jogam em diferentes posições (DUNCAN et al., 2006). As 
médias apresentadas pelos atletas das diferentes posições foram melhores que os resultados alcançados pelos jovens atletas de Juazeiro do Norte no percentual de gordura e nos testes de impulsão vertical e sentar e alcançar, exceto na posição de oposto, onde os atletas da Inglaterra apresentaram uma média de $19,3 \mathrm{~cm}$ contra 24, $2 \mathrm{~cm}$ do presente estudo. Entretanto, vale ressaltar que a faixa etária dos jogadores de voleibol investigados no estudo de Duncan et al. (2006) era de 16-19 anos, enquanto os jovens atletas de Juazeiro do Norte tinham entre 15 e 17 anos de idade.

As classificações nos testes da bateria PROESP-BR e do teste de Impulsão Vertical não vão de encontro à predisposição apontada pelo método dermatoglífico. A grande incidência de L, como já foi citado acima, é um indicador de predisposição à velocidade, e, no entanto, apenas 16,7\% dos sujeitos conseguiram bons resultados neste teste. Já a associação de A e L, encontrada na fórmula digital AL é indicativo da derivação de força para força potência, porém, quando comparados com o rendimento do teste de impulsão vertical em estudos semelhantes, os resultados obtidos pelos atletas sujeitos ao presente estudo foram sempre abaixo. Todavia, o fato de os atletas dos referidos estudos possuírem uma estatura maior, possivelmente, pode influir para a obtenção de melhores resultados. Dos atletas comparados, apenas os jogadores de futebol possuem uma estatura menor $(169,81 \pm 3,25 \mathrm{~cm})$ quando comparados com a média dos indivíduos aqui investigados $(175,67 \pm 8,48 \mathrm{~cm})$.

O gráfico a seguir apresenta os valores normatizados através da transformação dos resultados numéricos, de todas as variáveis (valor observado - menor valor observado)/(maior valor observado - menor valor observado), a fim de traçar o perfil total dos jovens atletas submetidos ao estudo.

FIGURA 3- Gráfico Radar Fernandes Filho - perfil dermatoglífico e características somatomotoras de jovens atletas de voleibol

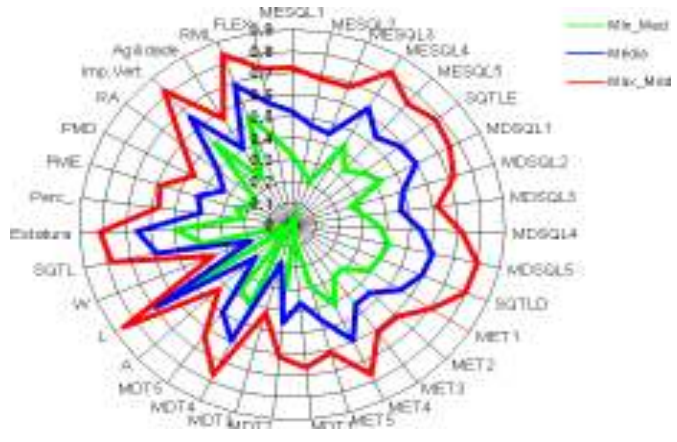

Nota: Mín_Méd = Mínima Média; Máx_Mád = Máxima Média 
O teste de correlação de Spearman não apontou correlações elevadas nas associações entre os ídices dermatoglíficos e as qualidades físicas básicas dos jovens atletas de voleibol submetidos ao presente estudo. Só foi encontrada uma correlação moderada na associação do arco (A) com a variável flexibilidade (ró $=0,587$ ). A literatura vigente no assunto (FERNANDES FILHO, 1997; SILVA DANTAS; FERNANDES FILHO, 2002; FERNANDES FILHO, et al., 2006) não relata tal associação, o que mostra que no presente estudo as características dermatoglíficas apontadas, com uma maior prevalência de L não justificam os resultados dos testes da bateria PROESP-BR.

\section{CONCLUSÕES}

O estudo mostra que o rendimento obtido nos testes aplicados para avaliar o nível das qualidades físicas básicas não condiz com a predisposição genética apontada pelo método dermatoglífico. A grande incidência do tipo de desenho L e a baixa intensidade de W, D10 e SQTL, apontam uma maior predisposição ao desenvolvimento da qualidade física velocidade, sendo que neste teste os jovens atletas obtiveram um dos piores resultados. Além disso, as correlações apontadas foram na sua maioria fracas. Ainda mais, quando comparados com atletas de equipes de nível estadual, nacional e estrangeiro, os sujeitos submetidos ao presente estudo apresentaram resultados inferiores.

Sabe-se que o desenvolvimento humano nos mais diversos aspectos, não depende somente de fatores hereditários como seus determinantes, mas também, de fatores fenotípicos. Aspectos ligados ao envolvimento com a prática são de suma importância para o desenvolvimento efetivo das capacidades físicas. É preciso ressaltar que na região onde foi desenvolvido o estudo, o esporte possui características predominantemente amadoras o que faz com que não haja um treinamento sistematizado e específico para o desenvolvimento e melhoria da performance dos atletas no que diz respeito às qualidades físicas exigidas para a prática do voleibol.

Sugere-se que sejam desenvolvidas futuras pesquisas com equipes que participem de um treinamento sistematizado e que busque desenvolver as valências físicas específicas do esporte praticado pelos sujeitos investigados, tentando assim melhor evidenciar as influências do fenótipo no desenvolvimento das qualidades físicas básicas. 


\section{REFERÊNCIAS}

ABRAMOVA, T. F.; NIKITINA, T. M.; OZOLIN, N. N. Possibilidades de utilização das Impressões Dermatoglíficas na seleção desportiva. Teoria e prática da Cultura Física. n.3, p. 10-15, 1995.

ALONSO, L. A. V.; SILVA DANTAS, P. M. Maturação, Dermatoglifia, Qualidades físicas e Atividade Física. em impressão, 2008.

BOELHOUWER, C.; BORGES, G. A. Aptidão física relacionada a saúde de escolares de 11 a 14 anos de Marechal Cândido Rondon - PR. Caderno de Educação Física: estudos e reflexões. Marechal Cândido Rondon, v. 4, n. 7, p. 19-30, 2002.

CABRAL, S. A. T.; CABRAL, B. G. A. T.; BEZERRA, F. P. Correlation between dermatoglyphic characteristics and explosive force in female under-17 volleyball players. FIEP Bulletin. Foz do Iguaçu, 2005.

CAStAnhede, A. L. K.; DANTAS, P. M. S.; FERnANDeS FILHO, J. Perfil dermatoglifico e somatotípico de atletas de Futebol de Campo masculino, de alto rendimento no Rio de Janeiro - Brasil. Fitness \& Performance Journal. Rio de Janeiro, v. 2, n. 4, p. 234-239, 2003.

CUMMINS, H.; MIDLO, CH. Palmar and plantar dermatoglyphics in primates. Philadelphia. 1942. p. 257

CUNHA, R. S. P.; FERNANDES FILHO, J. Identificação do perfi l dermatoglifico, somatotípico e das qualidades fisicas básicas da equipe brasileira feminina de esgrima. Fitness \& Performance Journal. v. 4, n. 1, p. 34-44, 2005.

CUNHA JÚNIOR, A. T.; FERNANDES FILHO, J. Caracteristicas dermatoglificas, somatotípicas, psicológicas e fisiológicas da seleção brasileira feminina adulta de handebol. Fitness e performance Journal. Rio de Janeiro, v. 5, n.2, p. 81-86, março/abril, 2006.

DANTAS, Estélio H. M. A prática da preparação física. $4^{\circ}$ ed. Rio de Janeiro: Shape, 1998.

DEL VILLAR, C. A. La preparación física del futbol basada en el atletismo. Madri: Gymnos, 1992.

DUNCAN, M. J.; WOODFIELD, L.; AL-NAKEEB, Y. Anthropometric and physiological characteristics of junior elite volleyball players. British Journal of Sports Medicine. n. 40, p. 649-651, 2006. doi: 10.1136/bjsm.2005.021998

FERNANDES FILHO, J.; DANTAS, P. M. S.; FERNANDES, P. R. Genética e treinamento esportivo: o uso prático da dermatoglifia. In: DaCosta, L. (Org.) Atlas do Esporte no Brasil. Rio de Janeiro: CONFEF, 2006.

FERNANDES FILHO J. A utilização de indices dermatoglificos na seleção de talentos. Revista Treinamento Desportivo. FMU, n. 2, p. 41-46, 1997. 
FILIN, V.; VOLKOV, V. Seleção de Talentos nos Desportos. Londrina: Midiograf, 1998.

FONSECA C. L. T.; DANTAS, P. M. S.; FERNANDES P. R.; FERNANDES FILHO J. Perfil dermatoglífico, somatotípico e da força explosiva de atletas da seleção brasileira de voleibol feminino. Fitness and Performance Journal.v. 7, n. 1, p. 35-40, 2008.

GABBETT, T. ; GEORGIEFF, B. Physiological and anthropometric characteristics of Australian junior national, state, and novice volleyball players. J Strength Cond Res. n. 21, v. 3, p. 902-8, 2007.

GAYA, A.; SILVA, G. (2007). Projeto Esporte Brasil. Manual de aplicação de medidas e testes, normas e critérios de avaliação. Porto Alegre: PROESP-BR, 2007.

GONÇALVES, H. R.; ARRUDA, M.; VALOTO, T. A.; ALVES, A. C.; SILVA, F. A.; FERNANDES, F. Análise de informações associadas a testes de potência anaeróbia em atletas jovens de diferentes modalidades esportivas. Arq. Ciênc. Saúde Unipar. Umuarama, v. 11, n. 2, p. 107-121, maio/ago. 2007.

GUEDES, D. P.; GUEDES, J. E. R. P. Crescimento, composição corporal e desempenho motor de crianças e adolescentes. São Paulo: C LR Balieiro, 1997.

GUEDES, D. P.; GUEDES, J. E. R. P.; BARBOSA, D. S.; OLIVEIRA, J. A. Aptidão física relacionada à saúde e fatores de risco predisponentes às doenças cardiovasculares em adolescentes. Revista Portuguesa de Ciências do Desporto. v. 2, n. 1, p. 31-46, 2002.

GONÇALVES, H. R. et al. Análise de informações associadas a testes de potência anaeróbia em atletas jovens de diferentes modalidades esportivas. Arq. Ciênc. Saúde Unipar. Umuarama. v. 11, n. 2, p. 107121, maio/ago. 2007.

JOÃO, A.; FERNANDES FILHO, J. Identificação do perfil genético, somatotípico e psicológico das atletas brasileiras de Ginástica Olímpica feminina de alta qualificação esportiva. Fitness \& Performance Journal; Rio de Janeiro, v. 1, n. 2, p. 12-20, 2002.

LOHMAN, T. G. Advanced in body composition assessment. Champaing, IL: Human Kinetics, 1992.

MASSA, M. et al., Análise de Referenciais Cineantropométricos de Atletas de voleibol masculino envolvidos em processos de promoção de talentos. Revista Mackenzie de Educação Física e Esporte. v. 2, n. 2, p. 101-113, 2003.

MEDEIROS, H. J.; ROCHA, V. M.; FERNANDES FILHO, J. Dermatoglifia e maturação. In: DANTAS, E. M.; FERNANDES FILHO, J. Atividade fisica e ciências da saúde. Rio de Janeiro: Shape, 2005.

MEDINA, M. F.; FERNANDES FILHO J. Identificação dos perfis genéticos e somatotípico que caracterizam atletas de Voleibol masculino adulto de alto rendimento no Brasil. Fitness \& Performance Journal; Rio de Janeiro, , v. 1, n. 4, p. 12-20, 2002. 
MOREIRA, R.; et al. Composição corporal de adolescentes da cidade de General Câmara - RS. Revista Perfil: Dossiê Projeto Esporte RS. 2005.

PÁVEL, D. A. C.; FERNANDES FILHO J. Identificação dos perfis dermatoglifico, somatotípico e das qualidades fisicas básicas de atletas de alto rendimento na modalidade de natação em provas de meiofundo e fundo. Fitness \& Performance Journal, v. 3, n. 1, p. 18-28, 2004.

PETROSKI, E. L.; et al. Antropometria: técnicas e padronizações. 3. ed. Blumenau: Nova Letra, 2007. PITANGA, F. J. G. Testes, medidas e avaliação em educação física. 3. ed. São Paulo: Phorte, 2004.

RÉ, A. H. N. et al. Interferência de características antropométricas e de aptidão fisica na identificação de talentos no futsal. Revista Brasileira de Ciência e Movimento. v.11, n. 4, p. 51-56, 2003.

ROLIM FILHO, N. G.; FERNANDES FILHO, J. Identificação do perfil dermatoglifico e somatotípico de pentatletas modernos brasileiros de alto rendimento. Revista de Educação Física. v. 139, p. 29-39, 2007.

RONQUE, Ê. V. Crescimento físico e aptidão física relacionada à saúde em escolares de alto nível socioeconômico. Dissertação (Mestrado)-Faculdade de Educação Física, Universidade Estadual de Campinas, Campinas, 2003.

FERNANDES, P. R.; FERNANDES FILHO, J. Dermatoglifia, somatotipia e consumo máximo de oxigênio em jogadores de futebol de campo, portadores e não portadores de paralisia cerebral. In: DANTAS, E. M.; FERNANDES FILHO, J. Atividade física e ciências da saúde. Rio de Janeiro: Shape, 2005.

DANTAS, P. M. S.; FERNANDES FILHO, J. Identificação dos perfis, genético, de aptidão física e somatípico que caracterizam atletas masculinos, de alto rendimento, participantes do futsal adulto, no Brasil. Fitness e Performance Journal, Rio de Janeiro, v. 1, n. 1, p. 28 - 36, 2002.

DANTAS, P. M. S. ; ALONSO, L. E.; FERNANDES FILHO, J. A dermatoglifia no futsal brasileiro de alto rendimento. Fitness e performance Journal, Rio de Janeiro, v. 3, n.3, p. 136 - 42, maio/junho, 2004.

SILVA. R. J. S. Características de Crescimento, Composição Corporal e Desempenho Físico Relacionado à Saúde em Crianças e Adolescentes de 07 a 14 Anos da Região do Cotinguiba (SE). Dissertação (Mestrado) - Universidade Federal de Santa Catarina, Florianópolis - SC, 2002.

THOMAS, J. R.; NELSON, J. K. Métodos de pesquisa em atividade fisica. 3. ed., Porto Alegre: Artmed, 2002.

TUBINO, M. G.; DACOSTA, L. Treinamento esportivo. In: DaCosta, L. (Org.) Atlas do Esporte no Brasil. Rio de Janeiro: CONFEF, 2006. 
TUCHE, W. et al. Perfil dermatoglífico e somatotípico de ciclistas de alto rendimento do Brasil. Revista de Educação Física. n. 132, p. 14-19, 2005.

ZARY, J. C. F.; FERNADEZ FILHO, J. Identificação do Perfil Dermatoglifico e Somatotípico dos Atletas de Voleibol Masculino Adulto, Juvenil e Infanto-Juvenil, de Alto Rendimento no Brasil. Revista Brasileira de Ciência e Movimento. v. 15, n. 1, p. 53-60, 2007. 


\section{HERMÓGENES GOMES SILVA}

Especialista em Fisiologia do Exercício e Treinamento Desportivo pela Universidade Castelo Branco UCB

\section{CÍCERO LUCIANO ALVES COSTA}

Curso de Licenciatura em Educação Física do Instituto Federal de Educação, Ciência e Tecnologia - IFET

\section{HUDDAY MENDES DA SILVA}

Curso de Licenciatura em Educação Física do Instituto Federal de Educação, Ciência e Tecnologia - IFET

\section{RICHARDSON DYLSEN DE SOUZA CAPISTRANO}

Curso de Licenciatura em Educação Física do Instituto Federal de Educação, Ciência e Tecnologia - IFET PROCIMH - Universidade Castelo Branco/Rio de Janeiro

\section{Referência do artigo:}

\section{ABNT}

SILVA, H. G. et al. Perfil dermatoglífico e qualidades físicas básicas de jovens atletas de voleibol. Conexões, v. 8, n.1, p. 1-15, 2010

\section{APA}

Silva, H. G., Costa, C. L. A., Silva, H. M., \& Capristano , R. D. S. (2010). Perfil dermatoglífico e qualidades físicas básicas de jovens atletas de voleibol. Conexões; 8(1): 1-15.

\section{VANCOUVER}

Silva HG, Costa CLA, Silva HM, Capristano RDS. Perfil dermatoglífico e qualidades físicas básicas de jovens atletas de voleibol. Conexões; 2010; 8(1): 1-15. 